\title{
Virgo status and commissioning results
}

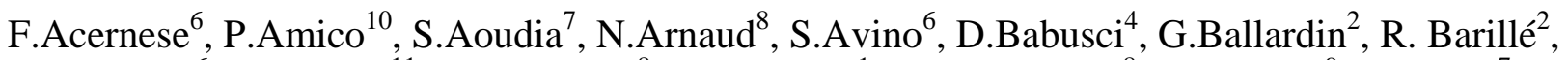

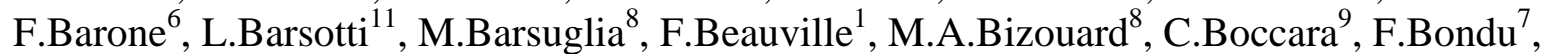
L.Bosi $^{10}$, C.Bradaschia ${ }^{11}$, S.Braccini ${ }^{11}$, A.Brillet ${ }^{7}$, V.Brisson $^{8}$, L.Brocco ${ }^{12}$, D.Buskulic ${ }^{1}$, E.Calloni ${ }^{6}$, E.Campagna ${ }^{3}$, F.Cavalier ${ }^{8}$, R.Cavalieri ${ }^{2}$, G.Cella ${ }^{11}$, E.Chassande-Mottin ${ }^{7}$, F.Cleva ${ }^{7}$, J.-P.Coulon ${ }^{7}$, E.Cuoco $^{2}$, V.Dattilo ${ }^{2}$, M.Davier ${ }^{8}$, R.De Rosa ${ }^{6}$, L.Di Fiore ${ }^{6 \ddagger}$, A.Di Virgilio ${ }^{11}$, B.Dujardin ${ }^{7}$, A.Eleuteri $^{6}$, D.Enard ${ }^{2}$, I.Ferrante ${ }^{11}$, F.Fidecaro ${ }^{11}$, I.Fiori $^{11}$, R.Flaminio ${ }^{1,2}$, J.-D.Fournier ${ }^{7}$, S.Frasca ${ }^{12}$, F.Frasconi ${ }^{2 ; 11}$, A.Freise ${ }^{2}$, L.Gammaitoni ${ }^{10}$, A.Gennai ${ }^{11}$, A.Giazotto ${ }^{11}$, G.Giordano $^{4}$, L.Giordano ${ }^{6}$, R.Gouaty ${ }^{1}$, D.Grosjean ${ }^{1}$, G.Guidi ${ }^{3}$, S.Hebri ${ }^{7}$, H.Heitmann ${ }^{7}$, P.Hello ${ }^{8}$, P.Heusse ${ }^{8}$, L.Holloway ${ }^{2}$, S.Kreckelbergh $^{8}$, P. La Penna ${ }^{2}$, V.Loriette ${ }^{9}$, M.Loupias ${ }^{2}$, G.Losurdo ${ }^{3}$, J.-M.Mackowski ${ }^{5}$, E.Majorana $^{12}$, C.N.Man ${ }^{7}$, F. Marchesoni ${ }^{10}$, F.Marion ${ }^{1}$, J.Marque ${ }^{2}$, F.Martelli ${ }^{3}$, A.Masserot ${ }^{1}$, M.Mazzoni ${ }^{3}$, L.Milano ${ }^{6}$, C. Moins ${ }^{2}$, J.Moreau ${ }^{9}$, N.Morgado ${ }^{5}$, B.Mours ${ }^{1}$, J.Pacheco ${ }^{7}$, A. Pai ${ }^{12}$, C.Palomba $^{12}$, F.Paoletti ${ }^{2 ; 11}$, S. Pardi ${ }^{6}$, A. Pasqualetti ${ }^{2}$, R.Passaquieti ${ }^{11}$, D.Passuello ${ }^{11}$, S. Peirani ${ }^{7}$, B.Perniola ${ }^{3}$, F. Piergiovanni ${ }^{3}$, L.Pinard ${ }^{5}$, R.Poggiani ${ }^{11}$, M.Punturo ${ }^{10}$, P.Puppo $^{12}$, K.Qipiani ${ }^{6}$,

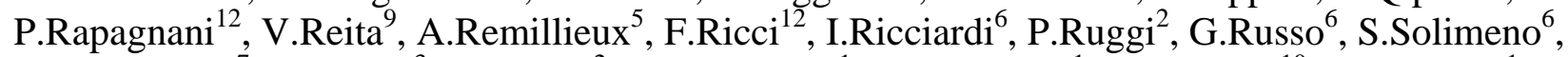
A. Spallicci ${ }^{7}$, R.Stanga ${ }^{3}$, R.Taddei ${ }^{2}$, D.Tombolato ${ }^{1}$, E.Tournefier ${ }^{1}$, F.Travasso ${ }^{10}$, D.Verkindt ${ }^{1}$,

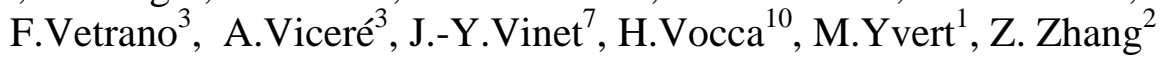

${ }^{1}$ Laboratoire d'Annecy-le-Vieux de physique des particules, Chemin de Bellevue - BP 110, 74941 Annecy-le-Vieux Cedex - France

${ }^{2}$ European Gravitational Observatory (EGO), Via E. Amaldi, I-56021 Cascina (PI) Italia

${ }^{3}$ INFN - Sezione di Firenze/Urbino, Via G.Sansone 1, I-50019 Sesto Fiorentino and/or Università di Firenze, Largo E.Fermi 2, I - 50125 Firenze, and/or Università di Urbino, Via S.Chiara, 27, I61029 Urbino, Italia

${ }^{4}$ INFN, Laboratori Nazionali di Frascati, Via E. Fermi, 40, I-00044 Frascati (Roma) - Italia

${ }^{5}$ SMA - IPNL 22, Boulevard Niels Bohr, 69622 - Villeurbanne- Lyon Cedex, France

${ }^{6}$ INFN - sezione di Napoli and/or Università di Napoli "Federico II", Complesso Universitario di Monte S. Angelo, Via Cinthia, I-80126 Napoli, Italia and/or Università di Salerno Via Ponte Don Melillo, I-84084 Fisciano (Salerno), Italia.

${ }^{7}$ Département Artémis - Observatoire Cote d'Azur, BP 42209, 06304 Nice, Cedex 4, France

${ }^{8}$ Laboratoire de l'Accélérateur Linéaire (LAL),IN2P3/CNRS-Université de Paris-Sud, B.P. 34, 91898 Orsay Cedex - France

${ }^{9}$ ESPCI - 10, rue Vauquelin, 75005 Paris - France

${ }^{10}$ INFN Sezione di Perugia and/or Università di Perugia, Via A. Pascoli, I-06123 Perugia - Italia

${ }^{11}$ INFN - Sezione di Pisa and/or Università di Pisa, Via Filippo Buonarroti, 2 I-56127 PISA - Italia

${ }^{12}$ INFN, Sezione di Roma and/or Università "La Sapienza", P.le A. Moro 2, I-00185, Roma

\footnotetext{
${ }^{\ddagger}$ Presented by L. Di Fiore for the Virgo Collaboration e-mail: luciano.difiore@na.infn.it
} 


\begin{abstract}
Virgo is a French-Italian collaboration for the construction and operation of a $3 \mathrm{~km}$ long interferometric gravitational wave (GW) antenna. The construction of the detector is already completed and the commissioning is quite advanced, while the data taking is expected to start in 2005. In this paper, we report on the present status of Virgo and on the results of commissioning activity. In particular, we analyse the first four engineering runs (C1-C4) and discuss the sensitivity obtained in C4.
\end{abstract}

\title{
1 - Introduction
}

The Virgo antenna is located in Cascina, a small city close to Pisa in Italy. A detailed description of the Virgo interferometer can be found in [1]; here we only summarize some relevant points. The detector is a $3 \mathrm{~km}$ long Michelson interferometer with Fabry-Perot cavities in the arms and power recycling. The light source is a $20 \mathrm{~W}$ Nd:YVO4 high power laser injection locked to a $1 \mathrm{~W} \mathrm{Nd:YAG}$ master laser [2]. Before entering the interferometer, the light beam is pre-filtered by a $144 \mathrm{~m}$ long mode cleaner, that is a triangular cavity with finesse $F=300$. All the main optical components of the interferometer are suspended to multi-stage seismic isolators [3] that allow extending the measurement band of the antenna down to a few Hz. The cavity mirrors that are the test masses of the detector are high homogeneity, low absorption monolithic fused silica mirrors, $20 \mathrm{~cm}$ in diameter and $10 \mathrm{~cm}$ in thickness, with losses al low as 5 ppm [4]. The whole optical path of the interferometer is in high vacuum, enclosed in a steel tube $1.2 \mathrm{~m}$ in diameter, with the payloads housed in ultra-high vacuum chambers. The dark fringe signal is filtered by a monolithic output mode cleaner (OMC) that filters out high order modes and aberrations.

The construction of the detector started in 1996. In 2000 INFN and CNRS settled the consortium EGO (European Gravitational Observatory), that has the objectives of supporting the commissioning of Virgo, its operation, maintenance and upgrades, creating and running a computing center for data analysis, ensuring site and infrastructure maintenance and promoting R\&D useful for the detection of gravitational waves. The construction of Virgo was completed in June 2003 with the installation of the last mirror at the end of the west arm of the interferometer; the antenna was inaugurated in July 2003 and the commissioning of the detector started in the same year.

\section{2 - The commissioning of Virgo}

The commissioning of many of the single components and sub-systems of Virgo was already performed during the operation of the central interferometer [5], so the commissioning of VIRGO has started by putting in operation the single FP cavities forming the $3 \mathrm{~km}$ long arms of the interferometer. The work was organized in the three different phases representing the major steps toward the operation of the full interferometer (Michelson with FP arms and power recycling): 
phase A - Independent Fabry-Perot cavities

phase B - Recombined Michelson interferometer

phase C - Recombined Michelson interferometer with power recycling

It is worth noticing that in phase A and B the power recycling mirror is misaligned by a few mrad so that the power recycling (PR) cavity is not resonating, As a consequence, the light entering the interferometer is very much reduced because the low transmission of PR mirror and the lack of recycling gain.

Until now, during the commissioning activity, there have been four engineering runs. The first two (C1, 14-17 November 2003 and C2, 20-23 February 2004) belong to the phase A relative to the commissioning of the single arms. In C1 only the north arm (NA) was operative, with the mirrors under local control for the angular degree of freedom, while in $\mathrm{C} 2$ the angular control was under linear alignment (LA) using Anderson technique for error signal extraction.

The third engineering run (C3, 23-27 April 2004) was split in two parts. It was partially in phase A, with the NA locked and under linear alignment as in $\mathrm{C} 2$, and with the addition of the socalled second stage of frequency stabilization (SSFS), that is the loop that stabilizes, in the measurement band of the interferometer, the laser frequency to the length of one interferometer arm.

The other part of the run was performed with the recombined Michelson interferometer with FP arms (but without LA and SSFS this was then the first run in phase B.

In the last run, (C4, 24-29 June 2004) the interferometer was operated in the recombined mode with LA and SSF (in this case the frequency reference was the common mode of the two FP cavities).

In figure 1, there are shown the sensitivities measured during the three phase A engineering runs. As we can see, the continuous upgrade of the detector improves progressively the sensitivity.

In figure 2, the sensitivity curves for the two phase B runs are reported, compared to the Virgo goal sensitivity. As we can see, despite the tremendous improvement in sensitivity from C1 to C4, the interferometer performance is still far from the design one [1, 6]. In the following section, it is described in some detail the interferometer configuration during $\mathrm{C} 4$, the different noise sources that limit the sensitivity are analysed and the strategy to reduce their effect and approach the design sensitivity are discussed.

\section{3 - The C4 engineering run.}

The C4 run lasted for 5 days (24-29 July 2004) with a rather high duty cycle; the longer continuous lock period was 28 hours and there where in total 9 losses of lock, never lasting more than a couple of hours. A schematic view of the interferometer configuration during C4 is reported in figure 3 . The ITF is operated as a Michelson with FP arms but without power recycling. The PR mirror is slightly misaligned so that only a few percent of the light entering the interferometer reaches the BS. The two FP arms are under linear alignment and the error signal is provided, by differential phase sensing, using quadrants looking at the light transmitted by the cavities. On the other side, the $\mathrm{BS}$ is, for the angular DOF, under local control. The laser frequency is stabilized, in the measurement band of the interferometer, with respect to the common mode motion of the FP arms. In low frequency, where there are the normal modes of the super attenuators, the frequency reference is provided by an ultra-stable rigid triangular reference cavity made of ULE; in this frequency band, the common mode of the FP cavities is controlled to hold them on resonance, the error signal being extracted by the reference cavity itself. The lock acquisition of the arms is obtained using the signals of photodiodes placed at the end of the arms and the one before the 
output mode cleaner (B1') for the dark fringe. When the interferometer is locked the dark fringe control is switched to the less noisy detector placed after the OMC (B1) while the differential and common mode of the FP cavities and BS are controlled looking at the light reflected by the interferometer.

In figure 4, it is reported the displacement sensitivity measured during $\mathrm{C} 4$, in the same plot, are reported the contributions of the identified noise sources that limit the performance of the interferometer. As we can see, for frequencies below $\sim 50 \mathrm{~Hz}$ the sensitivity is limited by BS control noise, both angular and longitudinal. The high angular noise is due to the fact that the BS is still under the rather noisy local control [7] and should be much lower when the LA will be implemented. The situation is a bit more complex for the longitudinal one. In this error signal, there are some peaks that have been identified as resonance modes of the input suspended bench (IB). The mechanism of transmission of this mechanical noise to the longitudinal control of the BS was not completely understood at the time of the conference. It as been recently shown that this is due to a mismatch between the modulation frequency used for phase sensing and the length of the IMC cavity. In principle, the modulation frequency should be equal to the free spectral range (FSR), so that, when the IMC is locked, both carrier and sidebands are resonating in the cavity. If they don't match, there is a direct coupling of IMC length variation, due to IB resonances, to the interferometer signals (a detailed analysis of this effect is beyond the scope of this paper). For example, for C4 it turned out that the mismatch was $70 \mathrm{~Hz}$ (or $1.5 \mathrm{~mm}$ in IMC total length). This effect could be reduced by actively controlling the total length of the IMC (or the nodulation frequency), but it is clear that the noise is so large that also the source of the noise needs to be reduced by decreasing the excitation, due to the bench control loops, of the IB normal modes, and perhaps by designing a new, more rigid bench.

Above $50 \mathrm{~Hz}$ and up to about $200 \mathrm{~Hz}$, the sensitivity is limited by DAC noise. This is the noise due to the limited dynamic range of the DACs used for feeding the coil drivers. A very important issue, in order to reduce this noise source, is the distribution of locking forces along the attenuator chain $[8,9]$. In order to cope with the limited ADC dynamics, it is necessary to split the actuation at different frequency bands acting in different points of the suspensions. The larger movements to be corrected (up to few $\mathrm{mm}$ ) are in the very low frequency and are mainly due to tides; this can be corrected by acting on the top stages of the SA inverted pendulums where there is a large dynamic range, while the out of band noise is effectively filtered out by the SA chain. Other large displacements are due to the SA normal modes that are confined in the frequency band from a few $\mathrm{mHz}$ to a few $\mathrm{Hz}$ and are only partially damped at the top stage by the "inertial damping" [9] . This part of the mirror movements, that can be as large as several hundred microns, can be controlled by acting on the marionetta, that is the last stage of the suspension where the test mass is hung. In this way, the extra noise is filtered by the last suspension stage (a $0.6 \mathrm{~Hz}$ oscillator). Above a few $\mathrm{Hz}$, the free running RMS motion of the mirrors is below one nm, and the action at this level, acting with the coils mounted on the reference mass, is only necessary for ensuring the stability of the control loop. During C4, the force distribution along the chain was only partially implemented (tidal control on the top stage) while all the remaining part was performed acting directly on the mirrors. For this reason, it was necessary to use coil drivers allowing to correct oscillations as large as about $20 \mu \mathrm{m}$. The reallocation of force to the marionatta should allow reducing the actuation range for the coils acting on the mirror to about one $\mathrm{nm}$ [9]. The contribution of this noise should then be reduced by more than four orders of magnitude, allowing the reaching of the low frequency sensitivity goal of Virgo. After the run, a lot of work has been devoted to the hierarchical control of Virgo and force reallocation to marionetta has been implemented with crossing frequency up to 10 $\mathrm{Hz}$, so we expect significant noise reduction for next engineering runs as soon as the coil driver strength will be reduced by some extent.

Above $200 \mathrm{~Hz}$, the sensitivity is mainly limited by photodiode electronic noise (B1) and laser frequency noise. Actually, this second noise is also limited by the electronic noise of the sensor used for the frequency control loop (B2). In the present configuration both photodiodes are 
illuminated with a power much lower than the one for which the electronics gain was designed. As already mentioned in the introduction, during C4 the PR mirror was misaligned by a few mrad, so that the PR cavity was not resonating and about $10 \%$ of the light power entering the ITF is transmitted to the BS. Furthermore, the lack of recycling gain gives a further reduction of the effective power in the interferometer by about a factor 50 with respect to the design. As a result, the detection is far from being shot noise limited. By implementing power recycling, the effect of electronic noise should be reduced by about three orders of magnitude, permitting to approach the shot noise limited design sensitivity above a few hundred Hz.

\section{4 - Discussion}

We have reported the main results of the commissioning of Virgo. In the last engineering run (C4) the interferometer was operated as a Michelson with FP arms and without power recycling. The observed sensitivity is still far from the design one, but all the main noise sources have been understood and strategies for reducing them at an acceptable level have been individuated. The first one is the reallocation of force to the marionetta in order to reduce the out-of-band noise due to the finite dynamics of coil drivers electronics and DAC. A second major improvement will be the transition to the so called phase $\mathrm{C}$ that is the operation of the ITF with power recycling. At the time of $\mathrm{C} 4$, the lock acquisition of the PR cavity was prevented because the light going back from the interferometer to the input mode cleaner was back scattered in the wrong direction (due probably to some defects in the IMC curved mirror) and entered again the interferometer, disturbing the control system error signals. Recently, the PR cavity has been locked, with a temporary solution, by replacing one of the mirrors of the input telescope with one with only $10 \%$ reflection. In this way, the light reflected to the IMC is reduced by two orders of magnitude, but at the cost of reducing by a factor of 10 the power inside the interferometer. A further improvement will be the insertion, between IMC and PR mirror of a Faraday Isolator (FI) that prevents light reflected from the interferometer from reaching the IMC; this requires a major change in the injection system, since the FI cannot be placed on the present IB.

For this purpose, it is presently under study a new input bench. The goal is not only the possibility of housing the FI and related optics, but more in general to get a more rigid setup without high Q resonances in the frequency band of VIRGO. The IB upgrade is planned sometime at the beginning of 2005, as soon as the design is completed.

In conclusion, after the improvement described here will be implemented, Virgo should reach a better sensitivity that allows starting scientific data taking, hopefully before the end of 2005.

\section{5 - References}

[1] - F.Acernese et al., Class. Quantum Grav. 21 (2004) S385-S394

[2] - F. Bondu et al., Class. Quantum Grav. 19 (2002) 1829-1833

[3] - S.Braccini et al., Class. Quantum Grav. 19 (2002) 1623-1629

[4] - F Beauville et al., Class. Quantum Grav. 21 (2004) S935-S945

[5] - F.Acernese et al., Astroparticle Physics 21 (2004) 1-22

[6] - F.Acernese et al., Astroparticle Physics 20 (2004) 617-628

[7] - http://www.virgo.infn.it/senscurve/ 
[8] - M.Barsuglia et al. VIR-NOT-NAP-1390-143, (2000)

[9] - G. Losurdo et al. Class. Quantum Grav. 19 (2002) 1631-1637. 


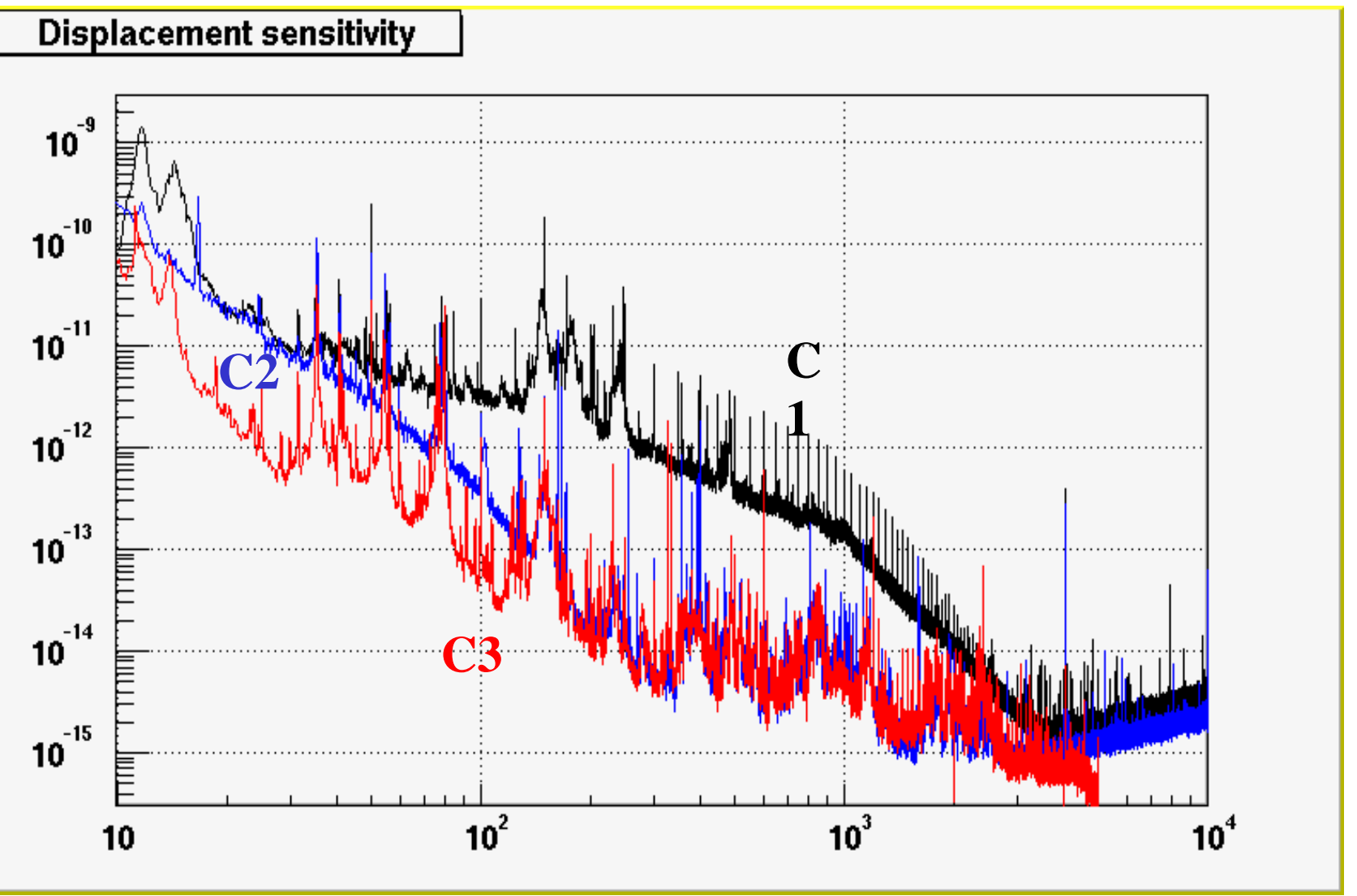

Figure 1 - Sensitivity $\left(\mathrm{m} / \mathrm{Hz}^{1 / 2}\right)$ of a single FP arm of VIRGO during the first three engineering runs (C1, C2 and C3). 


\section{Virgo: recombined interferometer sensitivity}

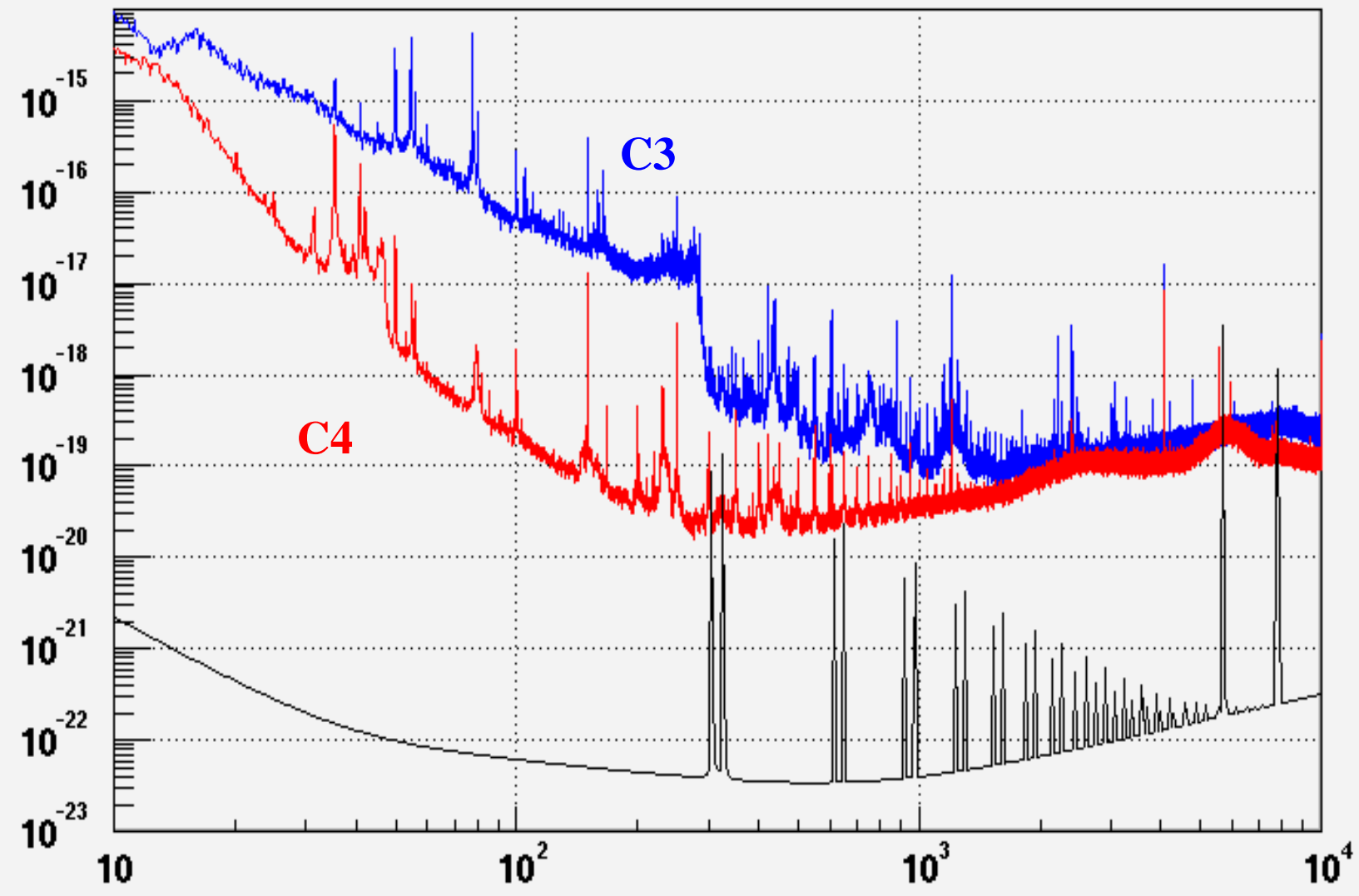

Figure 2 - h sensitivity $\left(1 / \mathrm{Hz}^{1 / 2}\right)$ of VIRGO (Michelson with $\mathrm{FP}$ arms) during the last two engineering runs (C3 and $\mathrm{C} 4)$, compared to the design sensitivity. 


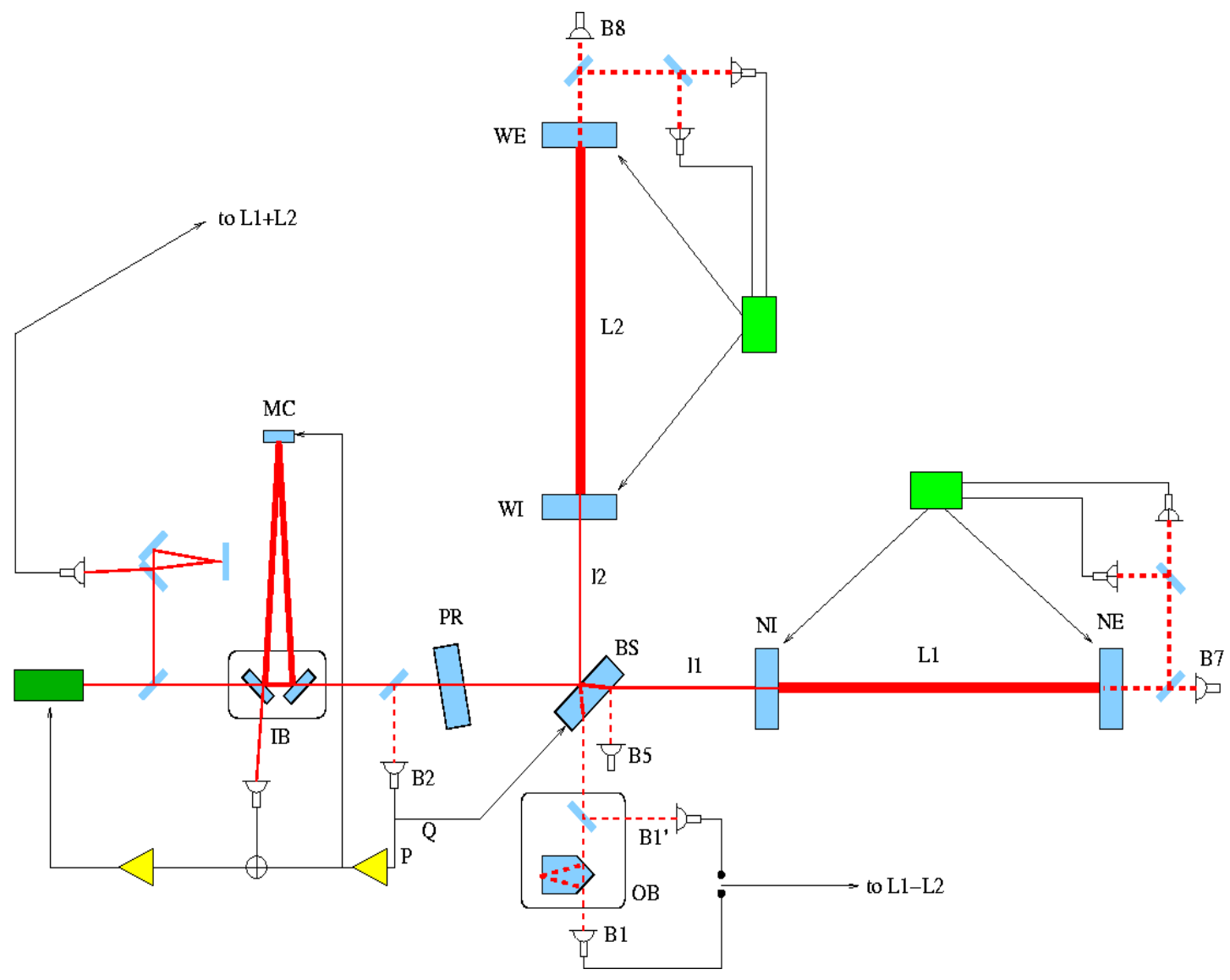

Figure 3 - Scheme of the Virgo interferometer during C4. The ITF is operated as a Michelson with FP arms but without power recycling. The PR mirror is slightly misaligned so that only a few percent of the light entering the interferometer reaches the BS. The two FP arms are under linear alignment, while the BS is for the angular DOF under local controls. The laser frequency is stabilized, in the measurement band of the interferometer with respect to the common mode motion of the PF arms. In low frequency, where there are the normal modes of the super attenuators, the frequency reference is provided by an ultra-stable rigid triangular reference cavity made of ULE; in this frequency band, the common mode of the FP cavities is controlled to hold them on resonance, the error signal being extracted by the reference cavity itself. 


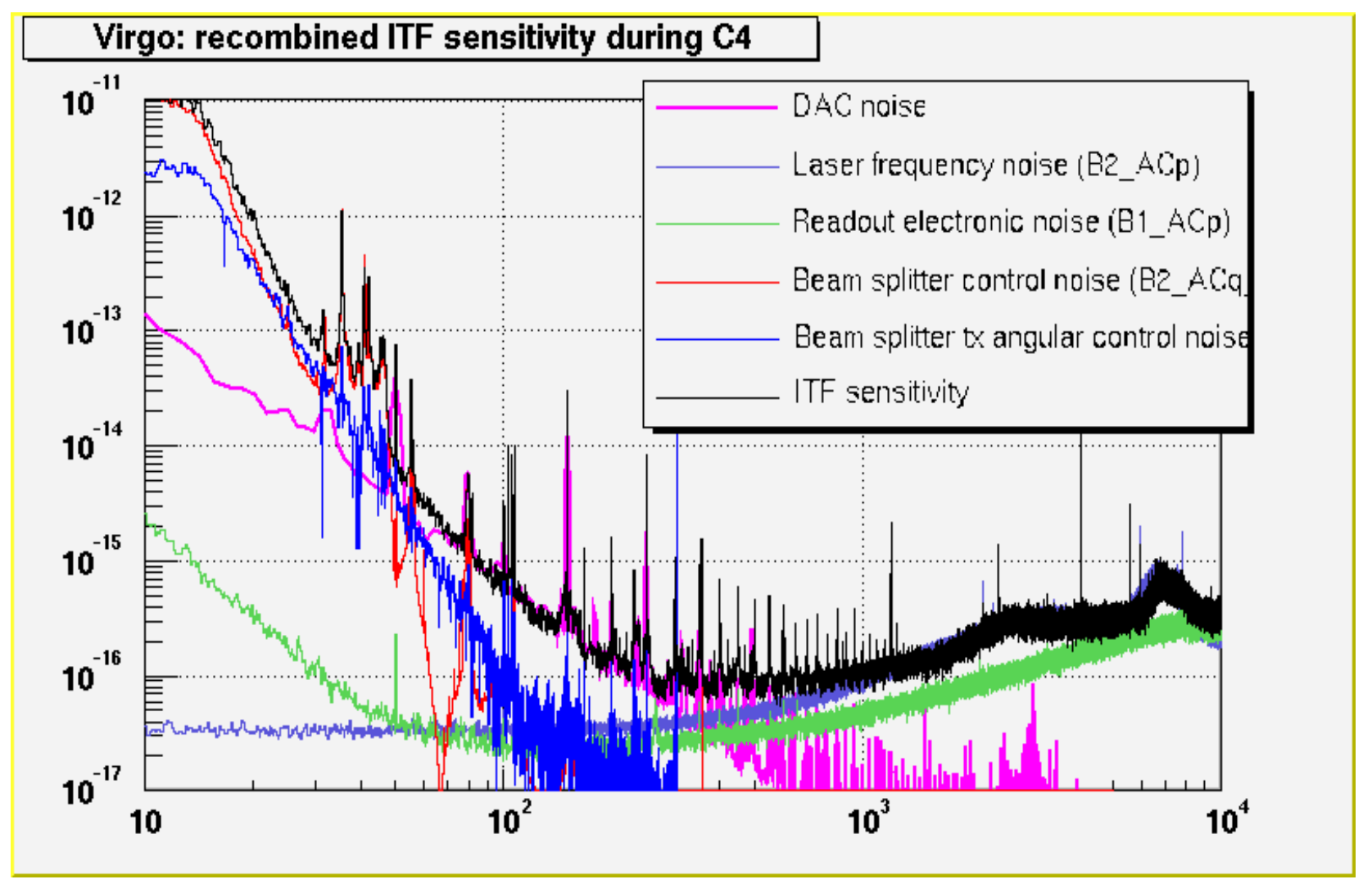

Figure 4 - Sensitivity curve $\left(\mathrm{m} / \mathrm{Hz}^{1 / 2}\right)$ of VIRGO (Michelson with FP arms) during the C4 engineering run. It is also shown the contribution of the various noise sources limiting the interferometer sensitivity at different frequencies. As we can see, the sensitivity is limited, below about $60 \mathrm{~Hz}$, by BS control noise, by ADC electronic noise between 60 and $300 \mathrm{~Hz}$ and by laser frequency noise and readout electronic noise above. The strategies for reducing these noise contributions in order to approach the design sensitivity are discussed in the text. 